\title{
Dental considerations in pregnancy
}

\begin{abstract}
Pregnancy is a unique state prompting a few physiological transient changes in the body frameworks including the oral depression. So as to keep up great oral wellbeing, the dental treatment ought not be retained. The dental management of pregnant patients includes uncommon contemplations. The dental management of pregnant patients requires extraordinary consideration. Dental specialists, for instance, may postpone certain elective techniques so they correspond with the times of pregnancy which are given to development versus organogenesis. At different occasions, the dental consideration experts need to adjust their ordinary pharmacological armamentarium to address the patients' needs versus the fetal requests. Applying the nuts and bolts of preventive dentistry at the essential level will expand the extent of the pre-birth care. Dental specialists ought to energize all the patients of the childbearing ages to look for oral wellbeing directing and assessments when they discover that they are pregnant. In this article we will tell how the dentist can prevent the diseases in pregnancy.
\end{abstract}

Keywords: pregnancy, erosion, trimester, gingivitis, dental caries
Volume 6 Issue 5 - 2020

\section{Shaik Ali Hassan}

Dental surgeon, Manav Rachna Dental College, India

Correspondence: Shaik Ali Hassan, Dental surgeon, Manav Rachna Dental College, Faridabad, Haryana, India, Email alishaikhassan@gmail.com

Received: September 09, 2020 | Published: October 23, 2020

\section{Introduction}

An expansion in the emission of the female sex hormones, estrogen by 10 crease and progesterone by 30 overlay, is significant for the typical movement of a pregnancy. ${ }^{1}$ The expanded hormonal discharge and the fetal development instigate a few foundational, just as nearby physiologic and physical changes in a pregnant lady. The principle foundational changes happen in the cardiovascular, haematologic, respiratory, renal, gastrointestinal, endocrine, and genitourinary frameworks. The nearby physical changes happen in various pieces of the body, which incorporate the oral depression. These aggregate changes may present different difficulties in giving dental consideration to pregnant patients. Accordingly, understanding the physiologic changes of the body and the impacts of the dental radiation and the prescriptions which are utilized in dentistry for the pregnant ladies, lactating moms and the babies, is basic for the administration of the pregnant and nursing moms. ${ }^{2}$

During pregnancy, ladies may encounter fundamental issues for example, respiratory changes: dyspnoea (in $60-70 \%$ of all the pregnant ladies), hyperventilation, wheezing, an upper ribcage breathing example and chest broadening andrhinitis; haemodynamic changes: rise of the coagulation factors V, VII, VIII, X and XII, and decrease of the components XI and XIII, with an expanded fibrinolytic action to make up for the expanded coagulating propensity; gastrointestinal modifications: an expanded intragastric pressure what's more, a decrease in the bring down oesophageal sphincter volume which is optional to hindrance of the creation of the motilin peptide hormone because of an ascent in progesterone fixations which are seen in this period - which offer ascent to indigestion (corrosiveness) in 30-70\% of the apparent multitude of pregnant ladies and a right around twooverlap prolongation of the gastric purging time when contrasted with those in non-pregnant ladies. ${ }^{3,4}$ Queasiness and regurgitating are experienced by $66 \%$ of the apparent multitude of pregnant ladies, beginning around 5 weeks after the last menstrual period, and arriving at a most extreme pervasiveness following 8-12 weeks. In this specific circumstance, the morning dental arrangements are to be dodged by pregnant ladies with an expanded spewing propensity because of pregnancy; renal changes: an expanded renal perfusion, especially during the subsequent half of the pregnancy, which offers ascend to an expanded medication discharge in the pee. Medication dosing changes are in this manner normally required in such patients. Endocrine changes are additionally watched in pregnant ladies: gestational diabetes is seen in $45 \%$ of all the pregnant ladies. Then again, the decubitus hypotension disorder or the vena cava condition is seen in the last phase of the pregnancy in around $8 \%$ of the apparent multitude of cases, as an aftereffect of a trouble in the venous come back to the heart, which is brought about by pressure of the mediocre vena cava by the gravid uterus. This condition shows as an unexpected drop in the blood pressure, with sickness, tipsiness and blacking out, when the patient is in the level position. ${ }^{5}$

\section{Dental diseases during pregnancy \\ Gingivitis}

Gum disease or draining delicate gums is the most widely recognized dental issue and adds to around $60-70 \%$ of pregnant ladies. Such conditions are basic because of diminished insusceptible reaction, hormonal variances of estrogens and progesterone and changes in typical oral flora. ${ }^{6,7}$

\section{Management}

Tributes to improve the condition may include proficient prophylaxis for example scaling, every day fluoridated tooth brushing, flossing and saline mouth flushes ought to be supported that may help in facilitating the aggravation. Moreover, chlorhexidine mouth washes may give included benefit. ${ }^{8-11}$

\section{Dental caries}

Honors to improve the condition may contain proficient prophylaxis for example scaling, day by day fluoridated tooth brushing, flossing and saline mouth flushes ought to be supported that may help in facilitating the aggravation. Likewise, chlorhexidine mouth flushes may give included benefit. ${ }^{12-14}$

\section{Management}

It is fitting for pregnant ladies to restrict sweet eating routine, brush consistently with fluoridated tooth glue and use over the counter fluoridated mouth washes to balance the impact of demineralization 
because of heaving. The part of effective uses of fluoride is all around acknowledged for the avoidance of caries. ${ }^{15,16}$ Fluoride delivering therapeutic materials, for example, glass ionomers can hinder optional caries also, medications, for example, methamphetamine that may additionally bother dental caries ought to be avoided..$^{17,18}$

\section{Periodontal disease}

About $30 \%$ of pregnant ladies experience the ill effects of periodontal diseases. ${ }^{19,20}$ While the function of raised degrees of circling estrogen is settled in higher commonness of gum disease and gingival hyperplasia during pregnancy, ${ }^{21}$ the affiliation among pregnancy and oral ailments like periodontitis require further exploration. The part of raised degrees of incendiary markers (for example interleukin 6, interleukin 8 and PGE2) has been found in the amniotic liquid of kid bearing ladies having periodontal conditions, which is viewed as related with untimely work and low birth weight. ${ }^{21-23}$

\section{Management}

Ongoing proof obviously exhibits that scaling and root arranging is viewed as sheltered during pregnancy and improves both maternal and neonatal health (21)..The management techniques to conquer periodontal illness in pregnant females grasps cautious finding by the dental medical care proficient, root arranging/profound scaling and recommending $0.12 \%$ day by day chlorhexidine mouth flushes to restrict the advancement of sickness. Chlorhexidine is arranged as FDA class B and estimated safe to rehearse in pregnant women. ${ }^{24}$

Tooth mobility: Because of hormonal surge mineral changes in lamina dura and aggravation in the periodontal tendon connection, influence portability of teeth prompting periodontal diseases..$^{25,26}$

Management: This condition can be made reversible whenever given remedial dosages of nutrient $\mathrm{C}$ alongside expulsion of neighborhood gingival aggravations.

Tooth erosion: Tooth erosion, another undesirable dental issue is thought of to be brought about by pregnancy actuated spewing. It is perceived that dental erosion can be successfully controlled with the utilization of an answer containing sodium bicarbonate that kills the corrosive and forestalls damages. ${ }^{27}$ It is encouraged to counsel patient's doctor and gastroenterologist to control the related clinical conditions.

Dental chair positioning: When performing seat side strategies it is vital to ensure that the pregnant patients are situated in the right and safe position. This assists with dodging any complexity, for example, recumbent hypotensive disorder in the dental seat. For instance, if a pregnant woman is situated in the prostrate situation, there are extraordinary odds of movement to medium hypoxemia and an anomalous blood vessel oxygen angle. Additionally there is a danger of pressure of the vena cava and aorta due to the gravid uterus which may prompt postural hypotension. Thusly it is significant that the managing dental specialist causes her to sit in the correct position; for example either situated with her right hip raised $10-12 \mathrm{~cm}$ with the goal that the weight on the vena cava is decreased or by putting the patient in a $5-15 \%$ tilt on her left side. In the event that the hypotension isn't alleviated, the patient ought to be approached to gain a full left horizontal position. These changes are anyway suggested during the third trimester. ${ }^{28-30}$

\section{Guidelines for dental management}

For the primary trimester (1-12 weeks): During the principal trimester, it is suggested that the patients be booked to evaluate their present dental wellbeing, to educate them of the progressions that they ought to expect during their pregnancies, what's more, to examine on the best way to evade maternal dental issues that may emerge from these changes. It isn't suggested that the strategies might be done as of now. The worry about doing strategies during the main trimester is twofold. To begin with, the creating kid is at a most serious danger which is presented by teratogens during organogenesis, and second, during the main trimester, it is known that upwards of one out of five pregnancies go through unconstrained premature births. Dental methods which are performed close to the time of an unconstrained fetus removal might be thought to be the reason, which lead to worries for both the patient and the professional, with respect to whether this could have been maintained a strategic distance from..$^{31,32}$

For the second trimester (13-24 weeks): Constantly trimester, the organogenesis is finished, and the danger to the baby is low. The mother has additionally had the opportunity to alter to her pregnancy, and the baby has not developed to a possibly awkward size that would make it hard for the mother to stay still for significant stretches. The situating of the pregnant patients is significant, particularly during the third trimester. As the uterus extends with the developing embryo and the placenta, it comes to lie legitimately over the mediocre vena cava, the femoral vessels, and the aorta. On the off chance that the mother is situated recumbent for the systems, the heaviness of the gravid uterus could apply enough strain to hinder a blood move through these significant vessels and to cause a condition which is called prostrate hypotension. In this condition, the circulatory strain drops auxiliary to the hindered blood stream, which causes an asyncopal or a close syncopal scene. This circumstance is handily cured by a legitimate situating of the patient on her left side and lifting the top of the seat, to keep away from pressure of the significant veins. The dental specialist ought not stop for a second to counsel the patient's obstetrician, should any question emerge about the security of a strategy, especially if there are exceptional conditions which are related with the pregnancy. ${ }^{33,34}$

The current suggestions are:

a. Oral cleanliness, directions and plaque control.

b. Scaling, cleaning and curettage might be performed in the event that they are fundamental.

c. The control of dynamic oral infections, assuming any.

\section{d. An elective dental consideration is protected}

e. Keep away from routine radiographs. Use specifically and when they are required.

For the third trimester (25-40 weeks): The fetal development proceeds and the focal point of the worry presently, is the danger to the up and coming birth measure and the wellbeing and solace of the pregnant lady (e.g the seat situating and the evasion of medications that influence the draining time). It is protected to perform a standard dental treatment in the early aspect of the third trimester, yet, from the center of the third trimester, routine dental medicines are stayed away from.

The current proposals are:

i. Oral cleanliness, guidelines and plaque control.

ii. Scaling, cleaning and curettage might be performed on the off chance that they are important.

iii. Stay away from an elective dental consideration during the second $50 \%$ of the third trimester.

iv. Stay away from routine radiographs. Use specifically and when they are required. 


\section{Radiographs, pregnancy and the hatchling}

$\mathrm{X}$-beams are a sort of electromagnetic radiation that have the capacity to ionize the material through which it passes. Ionizing living issue brings about a harm to the phones or the DNA. Contingent upon the measure of radiation and the phases of pregnancy, a harm to the fetal cells may bring about unsuccessful labors, birth deserts or mental debilitation. Anyway the dental radiation introduction of the embryo is insignificant. ${ }^{35}$ The incipient organism and the embryo, being significantly more radiosensitive than the grownup partner, are helpless to unfriendly impacts which result from the radiography presentation. During the initial fourteen days after the origination, the patient may have no information on being pregnant, along these lines making it reasonable for the doctor to ask about the last menstrual period before acquiring a radiographic picture. Since an overall addressing doesn't give an authoritative analysis about the pregnancy status, a lead protecting thought to be utilized for all the ladies who are in their childbearing a long time. The recurrence of changes and unfavorable impacts is straightforwardly identified with the portion, and the presentation is expanded when higher than should be expected radiation introductions are utilized to make up for the insufficient preparing quality. The presentation can likewise be expanded, contingent upon the view which is taken. The radiations from the maxillary foremost perspectives may go through the stomach zone, with entrance from the essential pillar, just as from the dissipate/radiation. Contingent upon the head position, a comparative presentation could likewise happen with the back perspectives. ${ }^{32,33}$ A few safeguards can be taken to stay away from the fetal presentation when radiographs should be taken. Utilizing a lead shield over the patient's midsection, utilizing an appropriately collimated bar, and utilizing a highspeed film, can diminish the fetal introduction. The teratogenicity of the radiation relies upon the fetal age and the portion of the radiation. The most serious danger to the embryo for teratogenicity and demise, is during the initial 10 days after the origination. The most basic time of the fetal advancement is between 4-18 weeks after the origination. The National Commission for Radiation Protective (NCRP) prescribes that the aggregate fetal introduction to radiation ought not surpass more than $0.20 \mathrm{~Gy}$, which can cause microcephaly and mental impediment.

\section{Conclusion}

Pregnancy is a remarkable period with different physiologic changes that help the development and development of another life. Each gestational ladies ought to be urged to look for clinical and dental consideration during pregnancy, as a disappointment in treating the creating issues influences the wellbeing of both the mother and the unborn youngster. The dental consideration experts must increase a fundamental comprehension of the fundamental physiological changes of pregnancy, the impacts which are identified with the utilization of prescriptions during incubation, and how these may cooperate with the conveyance of dental consideration. This understanding guides the improvement of the therapy plan and the conveyance of the vital clinical, wholesome and dental consideration, just as it readies the experts for directing their pregnant patients.

\section{Acknowledgments}

None.

\section{Conflicts of interest}

Author declare that there is no conflict of interest.

\section{Funding}

None.

\section{References}

1. Tracy M, Dellinger H. Mark Livingston. Pregnancy: Physiological changes and considerations for dental patients. Dent Clin N Am. 2006;50:677-697.

2. Lakshman Suresh, Lida Radfar. Pregnancy and lactation. Oral Surg Oral Med Oral Pathol. Oral Radiol Endod. 2004;97:672-682.

3. American Academy on Pediatric Dentistry Council on Clinical Affairs Committee on the adolescent. Guidelines on oral health care for the pregnant adolescent. Pediatr Dent. 2008-2009;30:102-106.

4. Turner M, Aziz SR. Management of the pregnant oral and maxillofacial surgery patient. J Oral Maxillofac Surg. 2002;60:1479-1488.

5. Dommisch H, Staufenbiel I, Schulze K, et al. Expression of antimicrobial peptides and interleukin-8 during early stages of inflammation: an experimental gingivitis study. J Periodont Res. 2015; 50(6):836-845.

6. Rashidi Maybodi F, Haerian-Ardakani A, Vaziri F, et al. CPITN changes during pregnancy and maternal demographic factors 'impact on periodontal health. Iran J Reprod Med. 2015;13:107-112.

7. Bajaj N, Tandon S. The effect of Triphala and Chlorhexidine mouthwash on dental plaque, gingival inflammation, and microbial growth. Int $J$ Ayurveda Res. 2011;2:29-36.

8. Rizvi A, Zafar MS, Farid WM, et al. Assessment of antimicrobial efficacy of MTAD, sodium hypochlorite, EDTA and chlorhexidine for endodontic applications: an in vitro study. Mid-East J Sci Res. 2014;21:353-357.

9. Amini H, Casimassimo PS. Prenatal dental care: a review. Gen Dent. 2010;58:176-180.

10. Newnham JP, Newnham IA, Ball CM, et al. Treatment of periodontal disease during pregnancy: a randomized controlled trial. Obstet Gynecol. 2009;114:1239-1248.

11. Silk H, Douglass AB, Douglass JM, et al. Oral health during pregnancy. Am Fam Physician. 2008;77:1139-1144.

12. Cheek TG, Gutsche BB. Maternal physiologic alterations during pregnancy. Anesth Obstet. 1993;3:1-17.

13. Giglio JA, Lanni SM, Laskin DM, et al. Oral health care for the pregnant patient. J Can Dent Assoc. 2009;75:43-48.

14. Zafar MS, Ahmed N. Therapeutic roles of fluoride released from restorative dental materials. Fluoride. 2015;48:184-194.

15. Zafar MS. Effects of surface pre-reacted glass particles on fluoride release of dental restorative materials. World Appl Sci J. 2013;28:457462 .

16. Livingston HM, Dellinger TM, Holder R. Considerations in the management of the pregnant patient. Special Care Dent. 1998;18:183188 .

17. Patil S, Thakur R, Madhu K, et al. Oral health coalition: knowledge, attitude, practice behaviours among gynaecologists and dental practitioners. $J$ Int Oral Health. 2013;5:8-15.

18. Tarsitano BF, Rollings RE. The pregnant dental patient: evaluation and management. Gen Dent. 1993;41:226-234.

19. Michalowicz BS, DiAngelis AJ, Novak MJ, et al. Examining the safety of dental treatment in pregnant women. J Am Dent Assoc. 2008;139:685695.

20. Toppenberg KS, Hill DA, Miller DP. Safety of radiographic imaging during pregnancy. Am Fam Physician. 1999;59:1813-1820. 
21. Nora JJ, Nora AH, Sommerville RJ, et al. Maternal exposure to potential teratogens. JAMA. 1967;202:1065-1069.

22. Gazal G, Fareed W, Zafar M. Effectiveness of gaseous and intravenous inductions on children's anxiety and distress during extraction of teeth under general anesthesia. Saudi J Anaesth. 2015;9:33-36.

23. Andrade SE, Gurwitz JH, Davis RL, et al. Prescription drug use in pregnancy. Obstet Gynecol. 2004;191:398-407.

24. Grahame-Smith DG, Aronson JK. Oxford textbook of clinical pharmacology and drug therapy. 1992.

25. Schroeder PL, Filler SJ, Ramirez B, et al. Dental erosion and acid reflux disease. Ann Intern Med. 1995;122:809-815.

26. Kurien S, Kattimani VS, Sriram RR, et al. Management of pregnant patient in dentistry. J Int Oral Health. 2013;5:88-97.

27. Bomford J, Ledger J, O'keeffe B, et al. Ciprofloxacin use during pregnancy. Drugs. 1993;45:461-462.
28. Chaveli Lopez B, Sarrion Perez MG, Jimenez Soriano Y. Dental considerations in pregnancy and menopause. J Clin Exp Dent. 2011;3(2):e135-144.

29. Nayak AG, Denny C, Veena KM. Oral health care considerations for the pregnant woman. Dent update. 2012;39(1):51-54.

30. Singh M. The pregnant dental patient. J Mass Dent Soc. 2012;60(4):3234.

31. Tracy MDellinger H. Mark Livingston. Pregnancy: Physiological changes and considerations for dental patients. Dent Clin $N \mathrm{Am}$. 2006;50:677-697.

32. Richards AG. Dental X-ray protection. Dent Clin North Am. 1968;631641.

33. ADA Council of Scientific Affairs. An update on radiographic practices: information and recommendations. J Am Dent Assoc. 2001;132:234 238 . 